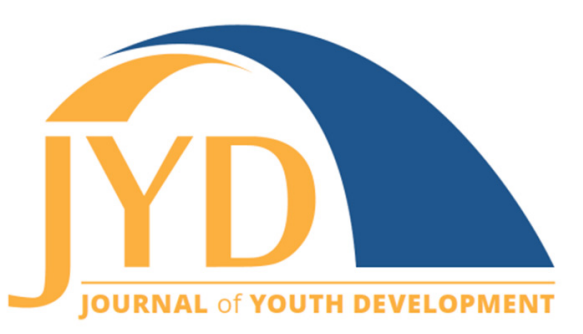

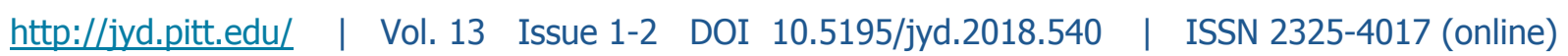

\title{
Young Adult Counselors with Diabetes at Diabetes Camps: The Effect of Being a Peer Mentor on Counselors' Health Behavior
}

\author{
Alison Stein Manning \\ Brown University, Departments of Pediatrics, Psychiatry and Child and Adolescent Psychiatry \\ alison.rs.manning@gmail.com \\ McLean Pollock \\ Duke University, Department of Psychiatry and Behavioral Sciences \\ mclean.pollock@duke.edu \\ Ben Clements \\ University of Vermont Medical Center \\ ben.clements@gmail.com \\ Elissa Furutani \\ Dana-Farber and Boston Children's Cancer and Blood Disorders Center, Harvard Medical School \\ elissa.furutani@gmail.com
}

Samuel Brotkin

Duke University, Department of Psychology and Neuroscience, Trinity College of Arts and Sciences samuel.brotkin@duke.edu

Joan Mansfield

Harvard Medical School; Joslin Diabetes Center

joan.mansfield@joslin.harvard.edu

Janis Kupersmidt

Innovation Research \& Training

jkupersmidt@irtinc.us

\section{Gregory Fritz}

Brown University, The Warren Alpert Medical School

gregory_fritz@brown.edu

\section{Gary Maslow}

Duke University, Department of Pediatrics, Department of Psychiatry and Behavioral Sciences gary.maslow@duke.edu

(cc) EY New articles in this journal are licensed under a Creative Commons Attribution 4.0 License. This journal is published by the University Library System, University of Pittsburgh and is cosponsored by the University of Pittsburgh Press. The Journal of Youth Development is the official peer-reviewed publication of the National Association of Extension 4-H Agents and the National AfterSchool Association. 


\section{Mentorship Effect on Counselors' Health Behavior}

\section{Abstract}

Adolescents and young adults (AYA) with type I diabetes (T1D) often struggle with illness management. Although diabetes camps have been shown to improve blood sugar control among campers, the effect of the camp experience on counselors' diabetes self-management has never been studied. In addition to the camp environment, it was hypothesized that peer factors among counselors, such as diabetes role modeling, would positively influence diabetes self-care behaviors and that counselors would be able to select diabetes role models based on these appropriate self-management behaviors. Counselors with T1D working at 2 summer camps were recruited to participate. Participants completed questionnaires including the Diabetes Behavior Rate Scale (DBRS) and a peer-assessment form in which participants were asked to nominate friends as diabetes role models, and to assign a role model score for each counselor. Hemoglobin A1C (HbA1c), a measure of blood sugar control, was obtained pre- and postcamp. Thirty-three participants completed the study. The average HbA1c score decreased $0.4 \%(\mathrm{p}<.01)$ over the 6 to 10 weeks of camp indicating improved metabolic control at camp. The number of nominations for diabetes role model was associated with diabetes self-care $(r=0.351, p=0.027)$. Diabetes role model scores were not associated with diabetes self-care $(r=0.272, \mathrm{p}=0.074)$. There was no correlation between HbA1c and diabetes self-care. The findings suggest that the camp counselor role is a potential target for intervention to promote positive outcomes for AYA with T1D. Lessons learned from how AYA with diabetes support one another at camp can be applied to community-based interventions for youth with T1D or other chronic illnesses.

Key words: type 1 diabetes, camp, self-management

\section{Introduction}

Since their inception in the 1920s, diabetes camps have become widespread with approximately 30,000 children attending diabetes camps in North America and over 16,000 more campers participating in one of the 180 diabetes camps throughout the rest of the world (American Diabetes Association, 2012). In addition, in 2010 there were an estimated 1,700 young adults with diabetes working at these camps as counselors (L. Abramson, personal communication, March, 2010). One of the goals of diabetes camping is to enable youth with diabetes to share their experiences with one another while becoming more personally responsible for their disease; this includes learning important lessons from counselors who themselves have diabetes (American Diabetes Association, 2006). Diabetes camps have been associated with improvements in diabetes-related knowledge, self-management skills and adherence, as well as self-esteem among campers (Maslow \& Lobato, 2009). Diabetes camps are often described as settings promoting Positive Youth Development (PYD). However, few studies have examined potential mechanisms by which attending diabetes camp could lead to growth, particularly in camp counselors.

Type 1 Diabetes (T1D) is a relevant chronic disease to study in the camp context, because it is a common condition affecting over 175,000 American youth under the age of 20 years with 
Mentorship Effect on Counselors' Health Behavior

increasing prevalence (Mayer-Davis et al., 2017). Diabetes management is complicated; it includes frequent testing of blood sugar levels, multiple daily insulin injections, constant dietary vigilance, and monitoring of physical activity. Adolescents and young adults (AYA) with T1D often struggle with these self-management tasks resulting in a decline in blood sugar control and increased risk for future disease-related complications (Bryden et al., 2001).

Adherence to self-management protocols declines during adolescence for a number of reasons. First, biological changes associated with puberty make blood sugar regulation more challenging. Second, psychologically, the developmental tasks of identity formation may cause adolescents to actively avoid self-care tasks to create an identity separate from their illness. In addition, the separation and individuation processes common to many emerging adults leads teens to distance themselves from parents who may have been primarily responsible for their disease management. The increased incidence of affective disorders in this age group is another factor that may impact motivation in regards to adherence. Third, socially, some adolescents may engage in risk-taking behaviors as they move more towards greater reliance on peers than on family (Karlsson, Arman, \& Wikblad, 2008). Finally, in addition to the biopsychosocial transitions common to all AYA, healthcare transitions are another hurdle for AYA with chronic illness. Studies have demonstrated that adherence declines significantly during the transition from pediatric to adult healthcare providers, as evidenced by a marked decrease in clinic attendance and increases HbA1c. Higher values of HbA1c indicate higher average blood sugars and poorer treatment adherence (Bryden et al., 2001; Kipps et al., 2002).

Peer relationships play an extremely important role in adolescence. Peer influence can have a positive or negative effect on treatment adherence (La Greca, Bearman, \& Moore, 2002). Peers may be more influential than diabetes educators, nurses, or doctors in helping youth manage the day-to-day difficulties of living with diabetes (Heisler, Vijan, Makki, \& Piette, 2010). Adults with T1D in a reciprocal peer support intervention had greater improvements in blood sugar management than those in the control group supported by nurse care managers, suggesting that peers may be a more powerful influence in promoting self-care than medical professionals (Heisler et al., 2010). In addition, peer leaders have been shown to be more effective than adults in delivering health education classes in schools (Mellanby, Rees, \& Tripp, 2000). Diabetes-specific social support from peers has been shown to be helpful in improving quality of life, and better quality of life has been associated with improved self-management (CassarinoPerez \& Dell'Aglio, 2015; Stahl-Pehe et al., 2017). 


\section{Mentorship Effect on Counselors' Health Behavior}

Diabetes camps take a strengths-based approach that is linked to the principles of PYD. PYD programs were initially developed to improve outcomes for at-risk youth, and the tenets of PYD have been applied to programming for youth with chronic illness. In the Lerner and Lerner model of PYD, there are five factors, the ' $5 \mathrm{Cs}^{\prime}$, that have been associated with positive development over time (Lerner et al., 2005). The 5Cs are Character, Connectedness, Caring, Competence, and Confidence (Lerner et al., 2005). Many diabetes camps strive to promote confidence and competence in counselors and campers alike through the modeling, learning, development, and practicing of new skills in an accepting environment that positively recognizes effective self-management behaviors (Johnson, Goldman, Garey, Britner, \& Weaver, 2011). Social connections are formed and maintained, and counselors develop empathy through their role in caring for younger campers. The three core components of effective PYD programming are skill building, opportunities for leadership, and sustained relationships with adults and peer mentors (Maslow \& Chung, 2013; Lerner et al., 2011), all of which are typically a part of diabetes camps. Clearly, opportunities for graduated leadership exist at camp; returning campers can transition to counselors-in-training (CITs), followed by promotion to junior and senior counselors, and possibly advancing into an administrative role. In the only other study examining counselors at diabetes camps, counselors reported that camp helped them learn leadership skills, effective diabetes management skills, and how to mentor and support children with T1D (Nicholl, Valenzuela, Nierenberg, \& Mayersohn, 2017).

The act of being a counselor may improve adherence behaviors in the counselors through peer relationships, specifically role modeling and peer mentorship. In a qualitative study on the experience of camp counselors at non-medical summer camps, Johnson et al. (2011) found that counselors believed that one of their primary jobs was to serve as role models to not only campers, but other counselors and staff. The effect of having role models at camp was qualitatively linked to enhanced adherence in counselors with T1D in a study by Nicholl et al: "I feel more motivated to manage my diabetes because my peers take such good care of their diabetes" and "the constant upkeep on checking blood glucose from the medical staff brushes off on me" (Nicholl et al., 2017, p.393). Qualitative data and anecdotal evidence suggest that youth select diabetes role models based on demonstrating appropriate self-care rather than personality or social status, but this hypothesis has not been empirically tested.

In summary, AYA with T1D are at higher risk for poor adherence and long-term complications than children or adults with T1D. Diabetes camps represent a unique environment in which participants can experience PYD through skill building, graduated leadership opportunities, and peer mentoring. One hypothesis is that camp provides an environment in which young adults 
Mentorship Effect on Counselors' Health Behavior

can serve as diabetes role models and help their peers develop in a positive direction both psychosocially and in regards to their medical self-care. In order to test this hypothesis, this study examined the question of whether or not AYA counselors with T1D identify appropriate diabetes role models who managed their diabetes effectively, as identified by self-management behaviors and a biological measure of blood sugar control.

\section{Methods}

\section{Participants}

All counselors and staff with T1D between 16 and 26 years of age were eligible to participate in the study that was conducted at two diabetes camps in New England. These staff were divided into four categories: Counselor in Training (CIT), Junior Counselor, Senior Counselor/Staff, and Administration. CITs were recent campers who had graduated to a position where they were learning to become full counselors. Senior counselors had more responsibility for a cabin group or activity than junior counselors and generally had prior experience working as a junior counselor. There were a few staff who did not work as directly with the campers (i.e. kitchen or maintenance staff), but had been at camp longer than campers and other staff, and they were included in the senior counselor group. The final category was administrative staff and included the camp director and other camp leaders. The sample for this study focused on counselors who had spent at least 6 weeks at camp.

Basic demographic data were collected as part of a pre-camp survey that assessed age, biological sex, years with diabetes, and prior experience with camp. Table 1 describes the study sample, which consisted of 33 participants between 16 and 24 years of age with an average age of 18.4 years. Most participants were male $(60 \%)$ and $40 \%$ were female. The sample included six CITs (18\%), 10 junior counselors (31\%), 13 senior counselors/staff (39\%), and four administrative staff (12\%). Most participants had attended the camp previously (87\%) with an average of 5 years. All participants had been diagnosed with diabetes at least 2 years previously. 
Journal of Youth Development | http://jyd.pitt.edu/ | Vol. 13 Issue 1-2 DOI 10.5195/jyd.2018.540 Mentorship Effect on Counselors' Health Behavior

Table 1. Demographic Data Regarding Diabetes Camp Counselors

\begin{tabular}{|l|l|}
\hline Demographic variable & Diabetes camp counselors $\mathbf{N}=\mathbf{3 3}$ \\
\hline Age in years mean (range) & $18.4(16-24)$ \\
\hline $\begin{array}{c}\text { Sex } \\
\text { Female }\end{array}$ & $13(40 \%)$ \\
\hline Role & $20(60 \%)$ \\
\hline CIT & 6 \\
\hline $\begin{array}{l}\text { Junior Counselor } \\
\text { Senior Counselor }\end{array}$ & 10 \\
\hline Administrative & 13 \\
\hline Number of years attending camp mean (range) & 5 years (0-14) \\
\hline Number of years with diabetes mean (range) & $9.5(2-21)$ \\
\hline
\end{tabular}

\section{Camp Program}

Each camp accommodated up to 75 campers per week; the majority of counselors at both camps had T1D. Counselors lived on-site throughout pre-camp training sessions and summer sessions, which extended over a total of 10 weeks. All counselors ate meals together with campers in the dining halls and spent at least 6 out of 7 days per week at camp activities.

\section{Measures}

The variables described below were collected from participants using pre- and post-camp surveys. The pre-camp survey included demographic data, medical history, and diabetes care behavior data. The post-camp survey included questions regarding medical issues that participants experienced at camp, and examined the primary outcomes related to two types of mentoring variables. 


\section{Mentorship Effect on Counselors' Health Behavior}

\section{Mentoring Variables}

The degree to which one of the AYA staff with diabetes was viewed by peers as a mentor was measured in two ways. A peer-assessment form was designed for this study and included an opportunity for participants to nominate mentors, to rank each of the other participants as a diabetes role model, and to list the characteristics of a diabetes role model.

\section{Mentor Nomination Variable}

Participants were asked to nominate up to three diabetes mentors. For each participant, the total number of nominations received was recorded. Some participants received no mentor nominations and others received over 5 . Receiving more mentor nominations was treated as an indicator of a participant being more strongly viewed as a diabetes mentor.

\section{Mentor Ranking Variable}

Participants were asked to rate each of the other participants on a 5-point rating scale ranging from a low of 1 (poor diabetes role model) to a high of 5 (excellent diabetes role model). The mean of ratings was calculated for each participant; a higher score was an indicator of the degree to which peers viewed him/her as a mentor.

Participants also listed characteristics of a peer diabetes role model, which were examined using qualitative analysis.

\section{Diabetes Medical Status}

As part of evaluating the medical status of each participant two types of data were collected pre- and post-camp including capillary blood samples and self-reported health behavior questionnaires. The blood sample was used to determine average blood sugar control by measuring HbA1c.

In addition to demographic information, the questionnaires included the Diabetes Behavior Rating Scale (DBRS), a measure of diabetes self-care. The DBRS is a self-administered, fixedchoice survey with good internal consistency and good reliability (Iannotti et al., 2006). The DBRS consists of 36 items and the score is reported as a proportion ranging from 0.06 to 1.0. A high score of 1.0 indicates consistent performance of all diabetes self-care behaviors and has been associated with better health outcomes in prior studies. 


\section{Procedure}

This study was approved by the Committee for Human Subjects at the Joslin Diabetes Center in Boston, Massachusetts. Counselors and staff were invited to participate in the study at the beginning of the camp season, which lasted between 6 and 8 weeks.

\section{Data Analysis}

First, prior to examining the mentoring outcomes, we examined changes in blood sugar control from pre-camp to post-camp using paired t-tests of HbA1c values.

Then, the primary outcomes of interest, mentor nominations and mean diabetes role model score, were assessed by examining correlations between the mentoring variables and the other demographic and medical variables, DBRS self-care score, role at camp, age, previous years at camp, and average blood sugar control as measured by HbA1c, using Pearson correlation coefficients.

Subsequently, the associations between number of diabetes role model nominations and average diabetes role model scores were compared with diabetes self-care scores and HbA1c using linear regression analysis. As a final step, multivariable linear regressions controlling for age were performed and one-way analysis of variance models were conducted to determine if mentor nominations, mean diabetes role model scores, DBRS self-care score, HbA1c post-test scores, age and years at camp differed by counselor and staff roles.

Thematic analysis was conducted on the qualitative data collected in response to the question, "What are the characteristics of a diabetes role model?"

\section{Results}

We compared the demographics for those who had complete data and those who had missing data and found no statistically significant differences between the two groups.

First, the change in blood sugar control while participants were at camp was assessed. The average $\mathrm{HbA1c}$ measurement at the beginning of camp was $8.1 \%$, and at the end of camp, the average score was $7.7 \%$, a decrease of $0.4 \%(p<0.01)$ over the 6 to 10 weeks of camp. An 
HbA1c goal of less than $7.5 \%$ is recommended for all pediatric age groups (American Diabetes Association, 2017). In looking at the bivariate correlation analyses, HbA1c was not associated with self-care or any other variable.

Table 2 compares the scores by counselor role on the two primary outcomes of mentor nominations and mentor rank score, as well as the mean diabetes self-care score, HbA1c posttest scores, and information regarding age and years at camp. For age, there was a statistically significant difference between roles $(p<.001)$, and a Tukey post-hoc test revealed that age was statistically significantly higher for senior counselors and administrators compared to counselors in training $(p<.001)$, senior counselors compared to junior counselors $(p=.001)$ and administrators compared to junior counselors $(p<.001)$, and administrators compared to senior counselors $(p<.001)$. However, there were no statistically significant differences by roles for mentor nominations, self-care scores, peer mentor scores, HbA1c post-test scores or years at camp.

Table 2. Outcomes (Mean Scores) by Camp Role

\begin{tabular}{|l|l|l|l|l|l|}
\hline & CIT & $\begin{array}{l}\text { Junior } \\
\text { Counselor }\end{array}$ & $\begin{array}{l}\text { Senior } \\
\text { Counselor }\end{array}$ & $\begin{array}{l}\text { Administrative } \\
(\mathbf{N = 4 )}\end{array}$ & $\begin{array}{l}\text { Overall mean } \\
\text { and (range) }\end{array}$ \\
\hline Mentor nominations & & 0.7 & 0.8 & 1.9 & 2.3 \\
\hline Self-care
\end{tabular}

+ Mentor Nominations is a count of the number of peer votes as a diabetes mentor ranging from 0 to 6 in this sample. \# Self-care is measured using the DBRS which ranges from a low of 0.06 to a high of 1.0 with higher scores indicating better self-management behaviors.

$¥$ Mentor Score is the mean of peer ratings on a 1-5 Likert scale of the degree to which a participant is a diabetes mentor with scores ranging from 1-5. 


\section{Mentorship Effect on Counselors' Health Behavior}

\section{Mentor Nomination Analysis}

On bivariate correlation analyses, there was a significant association between the number of nominations for diabetes role model and several other variables: diabetes self-care $(r=0.40$, $p=.021)$; role at camp $(r=0.40, p=0.02)$; age $(r=0.43, p=.012)$; and previous years at camp ( $r=0.36, p=.039)$. There was no association between blood sugar control (HbA1c) and mentor nominations.

A simple linear regression demonstrated that the self-care score could predict mentor nominations ( $\beta=6.6(2.72), p<0.05)$, accounting for $16 \%$ of the variability in mentor nominations. Adding age to the model slightly decreased the effect of self-care ( $\beta=5.65$ (2.55), $p<0.05)$, and both age and self-care accounted for $30 \%$ of the variability in mentor nominations.

\section{Mentor Ranking Analysis}

Peer mentor ranking scores were examined using the same procedure with bivariate correlation analyses followed by linear regression modeling. Similar to the model for mentor nomination, self-care scores significantly predicted peer mentor ranking scores ( $\beta=1.78(0.84), p<0.05)$, accounting for $12 \%$ of the variability in peer mentor scores. Age, however, did not have a statistically significant impact on this relationship.

The qualitative analysis revealed themes regarding characteristics of a diabetes role model including being knowledgeable about diabetes, having good self-management skills (good control, frequent checking of blood sugars and adjustment of insulin doses) and being healthy (adhering to diet, exercising). The idea also emerged of a diabetes role model as someone who helps others with diabetes management, teaches about diabetes, and encourages or motivates others with their diabetes self-care. Examples include: "A diabetes role model has good diabetes control, is enthusiastic about their diabetes care, and overall, sets a good example for how to manage their diabetes"; "Not only under control but have knowledge of the disease and share that knowledge with others to help them better their lives"; and ". . positive attitude with staff and campers, can teach by example." Only one person mentioned "good friend" as a quality of a diabetes role model. 


\section{Discussion}

This study aimed to examine the experience of AYA counselors and staff with T1D at summer camp and the degree to which they are identifying diabetes role models who are actually taking care of their diabetes effectively. The first key finding from this study was that AYA counselors demonstrated a significant improvement in blood sugar control as measured by HbA1c suggesting that something about the environment at diabetes camp may have promoted positive changes for AYA with T1D. Second, there was an association between being nominated as a diabetes mentor or having a higher diabetes mentor ranking score and better self-care behaviors. This suggests that AYA are able to accurately identify diabetes role models based on appropriate self-management. Given that peers are such an important influence in this age group, it is valuable to know that youth in this sample were able to identify peer role models based upon self-care. This sets the stage for future studies to examine the link between having a diabetes role model and exhibiting improved health behaviors and metabolic control.

The lack of correlation between peer mentor nominations/rank score and average blood sugar levels is interesting given that peer mentor nominations/rank score were associated with better self-care. One would expect that improved self-care would result in improved average blood sugar values, though there is some inconsistency regarding this relationship in the literature (Guo, Whittemore, \& He, 2011). The lack of relationship may also be related to the short-time frame or to the fact that camp is a setting with many other complex factors contributing to the change in HbA1c.

There are several limitations to this study. First, the sample size is small and relatively homogeneous. The participants who are counselors at diabetes summer camps are not representative of all AYA with T1D. They are a unique population who self-select to take on a leadership role in the community, perhaps because they attended camp themselves or have an interest in mentoring younger campers with T1D. They also likely differ from other young adults with T1D on important variables such as socioeconomic status, family support, level of education, and race/ethnicity. Over $60 \%$ of the participants were college students and the majority of the others were planning on attending college. Future studies should include larger, more geographically, ethnically, and socioeconomically diverse samples. A second limitation was the lack of a control group. There may have been other factors that accounted for the significant improvement in average blood sugar control apart from the camp experience such as increased physical activity during the summer or lack of school-related stress. The camp environment is also highly structured with scheduled times for blood sugar monitoring and 


\section{Mentorship Effect on Counselors' Health Behavior}

planned, portioned meals which facilitate self-care. Third, post-camp average blood sugar levels were not available for several counselors who left camp before 6 weeks and thus were not included in this study. Finally, it is not known if the improvement in blood sugar levels will be maintained when the AYA counselors leave the summer camp environment; thus, examining health outcomes using a long-term longitudinal design is needed.

If being a mentor and receiving mentoring contribute to positive changes in health behavior, then the mechanism of change needs to be tested. One hypothesis is that peer mentors serve as positive role models who are observed and imitated; an alternative hypothesis is that the quality of the relationship between the peer and the mentor is the mechanism that explains positive change. A third alternative is that these two mechanisms potentiate each other such that counselors imitate the behavior of peer mentors when they have a close, positive relationship and when they admire their peer's adherence to positive self-care suggesting an additive or even, multiplicative relationship. Future study will be needed to examine the ways in which mentoring relationships at diabetes camp may be related to behavior change.

Despite its limitations, this study has several strengths. To the best of our knowledge, this is the first study to formally examine the effects of diabetes camp on blood sugar control of AYA counselors with T1D. HbA1c was obtained by a blood test directly rather than relying solely on self-report making this finding more reliable. In addition, the duration of the study was six to 10 weeks, which is longer than most other camp studies of chronically medically ill youth, which typically last only one week.

The only other study of AYA counselors with T1D reported increased motivation for disease management after camp related to the influence of well-managed peers and having diabetes role models at camp. Specifically, counselor participants reported that observing staff members and campers manage their diabetes contributed to their own enhanced motivation (Nicholl et al., 2017). Data from our study suggest that counselors are, in fact, selecting diabetes role models based upon exemplary self-care behaviors rather than social factors; thus, these findings lend quantitative support to Nicholl's qualitative analysis.

Camp administrators are well versed in the role that camp plays in campers' positive development. An important finding from this study that adds to the literature on the positive impact of the camp experience is that camp plays an important role in the health, well-being, and leadership development of counselors as well as campers (Johnson et al., 2011). By providing opportunities for counselors to hone their roles as mentors and role models, 


\section{Mentorship Effect on Counselors' Health Behavior}

administrators could promote AYA health management as well as improve campers' self-care. An implication of the findings from this study is that pre-camp training for AYA counselors might focus on helping counselors define and understand the importance of their role as positive role models and mentors to campers. Variability in being a positive mentor suggests the need for standardized training and ongoing on-the-job support and/or supervision to help counselors to present a more uniform and potentially efficacious camp experience.

Consistent with workers in other types of positive youth development programs, counselors can better leverage their relationships with campers to achieve greater and more universal positive outcomes. As natural mentors in the context of a camp experience, counselors should be more intentional in their interactions with campers to support behavioral change in their peer counselors and campers alike. For camp directors, there is an opportunity to use the Lerner and Lerner (2011) PYD framework directly by planfully incorporating the three main features of PYD programs. This study provides an example of how to achieve this. Diabetes camp counselors first are leaders contributing to the program. Second they are learning practical skills related to helping others with diabetes, as well as how to manage their own diabetes. Third, this all takes place in the context of sustained youth-adult relationships.

The power of having youth return for several years to the same camp is important for camp directors to harness. This long-term relationship can be leveraged so that youth can gain skills and develop in ways that might not be possible without the longitudinal relationship. Camp directors can intentionally focus on increasing the opportunity for their adolescent participants, as well as for their staff to develop leadership and mentoring skills. Future studies could assess the effects of instituting formal counselor training about the value and skills associated with mentoring compared to counselor training-as-usual on the self-care practices and HbA1c of counselors and campers from pre- to post-camp participation. Challenges to this approach include the time and costs of implementing such training programs. The degree to which counselors are viewed as mentors by their peers or by their campers could be assessed as part of gathering information regarding counselor performance. This type of feedback could be used to identify high-performing counselors who could help other counselors develop stronger role modeling/mentoring skills which may be more cost effective.

Improving existing interventions targeting emerging adults is of vital importance given the decrease in adherence to recommended behavioral protocols among AYA and the potential for long-term health complications. Attendance at summer camp provides a unique opportunity to empower AYA using PYD approaches to improve their self-management and in doing so, 


\section{Mentorship Effect on Counselors' Health Behavior}

motivate other transition-aged youth to have better self-care through interactions with peer role models. Those who work in settings with AYA with chronic illness should be aware that camping programs can help facilitate individual growth and maturity, and illness self-management.

\section{Conclusion}

This study provides evidence supporting the value of diabetes summer camp for AYA with T1D. Significantly, this is the first study to examine the experience of summer camp on blood sugar control among counselors with T1D. This study highlights the potential benefits of counseling at specialty medical camps for AYA with chronic illness. Providers should encourage youth, especially those transitioning to adulthood, to participate in camps or other PYD programs, not only as a service opportunity, but also because it may benefit their health and the health of their peers. Being a mentor for AYA with T1D is more than just providing guidance, it requires doing the hard work of taking care of oneself. More studies are needed to understand the process of peer mentorship and role modeling at camp to be able to harness the power of positive peer influence on disease-related self-management and to generalize it to other settings.

\section{References}

American Diabetes Association. (2006). Diabetes care at diabetes camps. Diabetes Care, 29 [Supplement 1], S56-58.

American Diabetes Association. (2012). Diabetes management at camps for children with diabetes. Diabetes Care, 35 [Supplement 1], S72-75. doi:10.2337/dc12-s072

American Diabetes Association. (2017). 12. Children and Adolescents. Diabetes Care, 40 [Supplement 1], S105-S113. doi:10.2337/dc17-S015

Bryden, K. S., Peveler, R. C., Stein, A., Neil, A., Mayou, R. A., \& Dunger, D. B. (2001). Clinical and psychological course of diabetes from adolescence to young adulthood: a longitudinal cohort study. Diabetes Care, 24(9), 1536-1540.

Cassarino-Perez, L., \& Dell'Aglio, D. D. (2015). Health-related quality of life and social support in adolescents with type 1 diabetes. Spanish Journal of Psychology, 17, E108. doi:10.1017/sjp.2014.101

Guo, J., Whittemore, R., \& He, G. P. (2011). The relationship between diabetes self-management and metabolic control in youth with type 1 diabetes: an integrative review. Journal of Advanced Nursing, 6ス11), 2294-2310. doi:10.1111/j.1365-2648.2011.05697.x 
Journal of Youth Development | http://jyd.pitt.edu/ | Vol. 13 Issue 1-2 DOI 10.5195/jyd.2018.540

Mentorship Effect on Counselors' Health Behavior

Heisler, M., Vijan, S., Makki, F., \& Piette, J. D. (2010). Diabetes control with reciprocal peer support versus nurse care management: a randomized trial. Annals of Internal Medicine, 153(8), 507515. doi:10.7326/0003-4819-153-8-201010190-00007

Iannotti, R. J., Nansel, T. R., Schneider, S., Haynie, D. L., Simons-Morton, B., Sobel, D. O., . . Clark, L. (2006). Assessing regimen adherence of adolescents with type 1 diabetes. Diabetes Care, 29(10), 2263-2267. doi:10.2337/dc06-0685

Johnson, S. K., Goldman, J. A., Garey, A. I., Britner, P. A., \& Weaver, S. E. (2011). Emerging adults' identity exploration: Illustrations from inside the "Camp Bubble". Journal of Adolescent Research, 26(2), 258-295. doi:10.1177/0743558410376832

Karlsson, A., Arman, M., \& Wikblad, K. (2008). Teenagers with type 1 diabetes--a phenomenological study of the transition towards autonomy in self-management. International Journal of Nursing Studies 45(4), 562-570. doi:10.1016/j.jinurstu.2006.08.022

Kipps, S., Bahu, T., Ong, K., Ackland, F. M., Brown, R. S., Fox, C. T., . . Dunger, D. B. (2002). Current methods of transfer of young people with Type 1 diabetes to adult services. Diabetic Medicine, $19(8), 649-654$.

La Greca, A. M., Bearman, K. J., \& Moore, H. (2002). Peer relations of youth with pediatric conditions and health risks: promoting social support and healthy lifestyles. Journal of Developmental and Behavioral Pediatrics, 23(4), 271-280.

Lerner, R. M. (2011). Positive youth development: Processes, programs, and problematics. Journal of Youth Development, 6(3), 1-25.

Lerner, R. M., \& Lerner, J. V. (2011). Advances in child development and behavior. In R. M. Lerner, J. V. Lerner, \& J. B. Benson (Eds.), Positive youth development, (Vol. 41, pp. 2-415). London, UK: Elsevier.

Lerner, R. M., Lerner, J. V., Almerigi, J., Theokas, C., Phelps, E., Gestsdóttir, S., . . von Eye, A. (2005). Positive youth development, participation in community youth development programs, and community contributions of fifth grade adolescents: Findings from the first wave of the 4-H Study of Positive Youth Development. Journal of Early Adolescence, 25(1), 17-71. doi: $10.1177 / 0272431604272461$

Maslow, G. R., \& Chung, R. J. (2013). Systematic review of positive youth development programs for adolescents with chronic illness. Pediatrics, 131(5), e1605-1618. doi:10.1542/peds.2012-1615

Maslow, G. R., \& Lobato, D. (2009). Diabetes summer camps: history, safety, and outcomes. Pediatric Diabetes, 10(4), 278-288.

Mayer-Davis, E. J., Lawrence, J. M., Dabelea, D., Divers, J., Isom, S., Dolan, L., . . Wagenknecht, L. (2017). Incidence trends of type 1 and type 2 diabetes among youths, 2002-2012. New England Journal of Mededicine, 376(15), 1419-1429. doi:10.1056/NEJMoa1610187 
Journal of Youth Development | http://jyd.pitt.edu/ | Vol. 13 Issue 1-2 DOI 10.5195/jyd.2018.540 Mentorship Effect on Counselors' Health Behavior

Mellanby, A. R., Rees, J. B., \& Tripp, J. H. (2000). Peer-led and adult-led school health education: a critical review of available comparative research. Health Education Research, 15(5), 533-545.

Nicholl, M. C., Valenzuela, J. M., Nierenberg, B., \& Mayersohn, G. S. (2017). Diabetes camp counselors: An exploration of counselor characteristics and quality of life outcomes. The Diabetes Educator, 43(4), 378-387. doi:10.1177/0145721717717246

Stahl-Pehe, A., Landwehr, S., Lange, K. S., Bachle, C., Castillo, K., Yossa, R., . . Rosenbauer, J. (2017). Impact of quality of life (QoL) on glycemic control (HbA1c) among adolescents and emerging adults with long-duration type 1 diabetes: A prospective cohort-study. Pediatric Diabetes. doi:10.1111/pedi.12487 